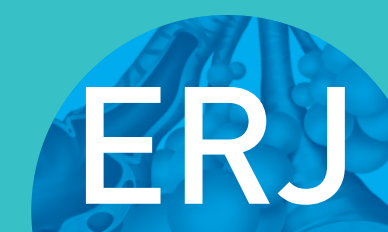

open research
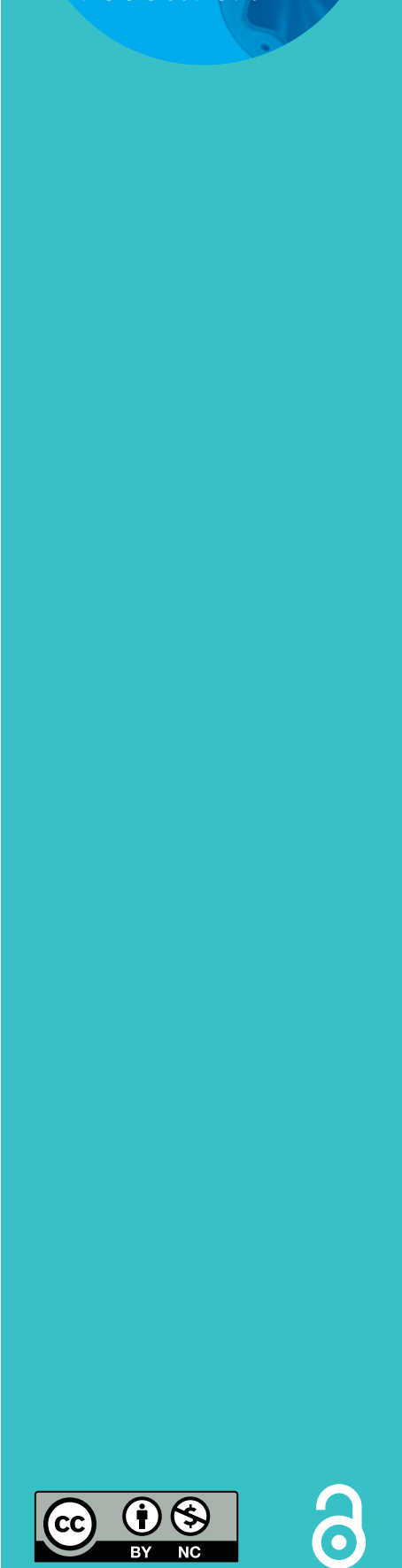

\section{latrogenic bleeding during flexible bronchoscopy: risk factors, prophylactic measures and management}

\author{
Maurizio Bernasconi ${ }^{1}$, Coenraad F.N. Koegelenberg ${ }^{2}$, Angela Koutsokera ${ }^{1}$, \\ Adam Ogna ${ }^{1}$, Alessio Casutt ${ }^{1}$, Laurent Nicod ${ }^{1}$ and Alban Lovis ${ }^{1}$
}

Affiliations: ${ }^{1}$ Division of Pulmonology, Dept of Medicine, University Hospital of Lausanne, Lausanne, Switzerland. ${ }^{2}$ Division of Pulmonology, Dept of Medicine, Stellenbosch University and Tygerberg Academic Hospital, Cape Town, South Africa.

Correspondence: Maurizio Bernasconi, Dept of Respiratory Medicine, Centre Hospitalier Universitaire Vaudois, University Hospital of Lausanne, Rue du Bugnon 21, 1011 Lausanne, Switzerland. E-mail: mauribernasconidgmail.com

ABSTRACT Significant iatrogenic bleeding during flexible bronchoscopy is fortunately rare and usually self-limiting. Life-threatening bleeding, however, can occur, especially after conventional or cryoprobeassisted transbronchial biopsy. The aim of this review is to provide the practising pulmonologist with a concise overview of the incidence, severity and risk factors for bleeding, to provide sensible advice on prophylactic measures and to suggest a plan of action in the case of significant bleeding. Bronchoscopy units should have a standardised approach and plan of action in the case of life-threatening haemorrhage. Wedging the bronchoscope in the bleeding segment, turning the patient in an anti-Trendelenburg position and onto the side in order for the bleeding lung to be in the dependent position, installing vasoconstrictors and using a tamponade balloon early are the recommended first-line strategies. Involving a resuscitation team should be considered early in the case of massive bleeding, desaturation and haemodynamic instability.

$@$ ERSpublications

Iatrogenic bleeding during flexible bronchoscopy http://ow.ly/w9Fy30bsoe5

Cite this article as: Bernasconi M, Koegelenberg CFN, Koutsokera A, et al. Iatrogenic bleeding during flexible bronchoscopy: risk factors, prophylactic measures and management. ERJ Open Res 2017; 3: 00084-2016 [https://doi.org/10.1183/23120541.00084-2016].

Received: July 202016 | Accepted after revision: March 182017

Conflict of interest: None declared.

Copyright $\odot$ ERS 2017. This article is open access and distributed under the terms of the Creative Commons Attribution Non-Commercial Licence 4.0. 


\section{Introduction}

Flexible bronchoscopy (FB) is one of the most commonly performed procedures by chest physicians. Iatrogenic bleeding, although rarely reported [1], is by far one of the most frightening situations encountered in daily practice [2]. Despite the fact that this complication may potentially be life-threatening $[3,4]$, the guidelines of the main thoracic societies on FB provide few recommendations on the management of iatrogenic bleeding [5]. The aim of this review is to provide the practising pulmonologist with a concise overview of the incidence, severity and risk factors for bleeding, to provide sensible advice on prophylactic measures and to suggest a plan of action in the case of significant bleeding.

References for this review were identified through searches of PubMed for articles published on this topic. Articles resulting from these searches and relevant references cited in those articles were reviewed.

\section{Incidence and severity of bleeding}

Bleeding during diagnostic FB occurs in between $0.26 \%$ and $5 \%$ of cases depending on the definition, the patient population and the procedures performed [1,6-8]. Transbronchial lung biopsies (TBLBs) increase the risk of bleeding significantly. Massive bleeding and mortality are exceedingly rare, and are predominantly induced by therapeutic rather than diagnostic bronchoscopies (incidence $0.059 \%$ versus $0.031 \%$ and $0.012 \%$ versus $0.003 \%$, respectively) [3]. However, all endoscopy centres, and particularly high-volume endoscopy units, may experience near-fatal or fatal bleeding during everyday practice [3].

The severity of bleeding has been characterised according to the volume of blood and fluid aspirated [1] or according to the intervention needed in order to control the bleeding [8]. The latter is less influenced by measurement variability and blood dilution with bronchial secretions and instilled substances [2], and so is more appropriate for describing clinically relevant bleeding. Moderate and severe bleeding (i.e. clinically significant bleeding) are defined as bleeding requiring wedging of the biopsied segment with the bronchoscope and as bleeding needing additional interventions (placement of a temporary bronchus-blocker, application of a fibrin sealant, admission to a critical care unit or the need for blood products), respectively [8].

\section{Risk factors}

Several factors have been reported to influence the risk of bleeding during FB, which can arbitrarily be divided into procedural related, patient related, haemostatic risk factors and related to the tissue sampled (figure 1). The risk of bleeding is expected to rise in the presence of multiple risk factors.

\section{Risk factors related to the endoscopic procedure}

Bleeding risk differs depending on the procedure performed and the vascularisation of the tissue sampled. The estimated risk for different procedures, inferred from the available literature and pulmonologists' appraisal [9], is summarised in figure 1. Inspection and bronchoalveolar lavage (BAL) are considered very atraumatic and have been reported to be safe, even via the transnasal route in severely thrombocytopenic patients [10, 11]. Many authorities, however, recommend oral flexible bronchoscope insertion in thrombocytopenic patients. Endobronchial ultrasound-guided transbronchial needle aspiration (EBUS-TBNA) is a very safe procedure, with bleeding occurring in only 3 out of 1317 patients undergoing the procedure $[12,13]$. In the same study, TBLB was the only factor associated with complications [13]. EBUS-TBNA may even be safe in patients taking clopidogrel [14]. In general, brushing and TBNA of peripheral and endobronchial lesions are very safe. Significant bleeding may occur after sampling of a very well vascularised tumour (e.g. carcinoid tumours) or after endobronchial biopsy of an endobronchial tumour (bleeding from a central lesion). Transbronchial biopsies of peripheral lesions or lung parenchyma (bleeding
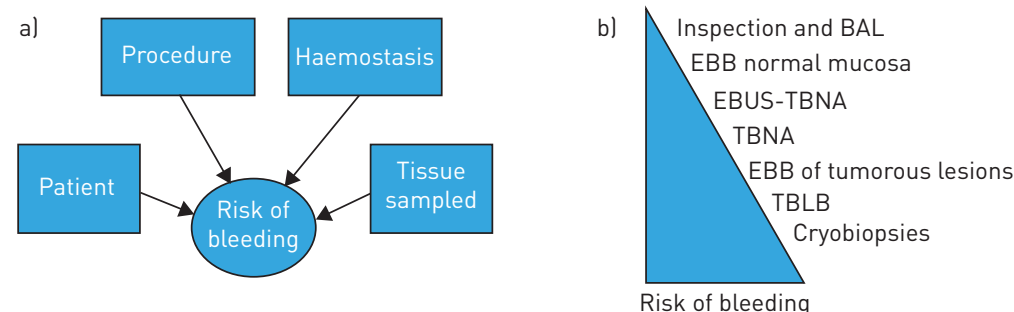

FIGURE 1 a) Factors influencing the risk of bleeding. b) Risk of bleeding for different procedures. Risk of bleeding is given as an estimated risk inferred from the available literature and according to the authors experience. BAL: bronchoalveolar lavage; EBB: endobronchial biopsy; EBUS: endobronchial ultrasound; TBNA: transbronchial needle aspiration; TBLB: transbronchial lung biopsy. 
from the periphery) are associated with the highest risk of clinically significant bleeding $[1,7,15]$. In fact, clinically moderate to severe bleeding was reported in about $1.1-2.8 \%$ of patients undergoing TBLB $[1,8,16]$. The highest risk of bleeding has been reported after cryobiopsies of lung parenchyma, which has recently been suggested as a technique allowing a higher diagnostic performance in interstitial lung disease [17-19]. This technique allows much larger biopsies than conventional TBLB, at the price of a trend towards a higher frequency of significant bleeding [19-21], motivating the preventive use of a tamponade balloon, which is inflated at the moment of biopsy [19]. An occlusion balloon is commonly introduced via the endotracheal tube and placed at the entrance to the lung segment from which the transbronchial lung biopsy is to be obtained, and inflated just after biopsy [21].

\section{Patient-related risk factors}

Several patient characteristics have been reported to influence the risk of bleeding during a diagnostic FB. Lung malignancy is a common indication for FB and has been consistently reported as a considerable risk factor for bleeding [1]. Immunocompromised patients [22], and especially lung transplant (LTX) recipients, are more likely to have new or worsened haemoptysis and to have the procedure terminated early for bleeding [23]. However, no specific risk factors for bleeding in LTX recipients could be found [24]. FB and tissue sampling in patients presenting a superior vena cava syndrome has been reported to be safe [25]. Pulmonary hypertension (PH) could, at least theoretically, lead to excessive bleeding following TBLB, and current recommendations [5] suggest that TBLB should be performed with caution in patients with elevated pulmonary arterial pressure (PAP) [5]. Furthermore, a survey has reported that most pulmonologists consider a mean PAP (mPAP) greater than $40 \mathrm{mmHg}$ as unsafe for TBLB [26]. In a prospective controlled trial in patients with $\mathrm{PH}$ from different aetiologies, 24 patients with a mean mPAP of $45 \mathrm{mmHg}$ underwent transbronchial biopsy. Even patients with severe $\mathrm{PH}$ experienced no significant bleeding or worsening hypoxaemia, suggesting that FB can be performed safely in patients with mild to moderate $\mathrm{PH}[27]$.

Patients with lung malignancy often have a procoagulatory state that may promote the development of venous thrombosis and lung embolism. It is therefore quite common to have to perform a bronchoscopy in a patient with a lung mass and a lung embolism. In haemodynamically stable and non-hypoxaemic patients, bronchoscopy and tissue sampling may be performed after withholding the anticoagulation therapy, but is considered a situation at high risk of bleeding and thrombotic complication (especially in the first 3 months of anticoagulation). In this situation, it is common practice to switch to low-molecular-weight heparin (LMWH) or unfractionated heparin (UFH) and omit subcutaneous LMWH the day of the procedure [5] or stop intravenously UFH $6 \mathrm{~h}$ before the procedure [5]. The anticoagulation is restarted $4 \mathrm{~h}$ after the procedure in the absence of bleeding.

\section{Haemostasis-related risk factors}

The goal of performing coagulation studies is to unmask disorders that could increase the risk of iatrogenic bleeding. However, the prevalence of inherited coagulopathies is low and, in patients with the most common disorder (von Willebrand's disease), the results of routine coagulation tests may be normal. Similarly to coagulation studies in patients undergoing elective surgery [28], coagulation tests before FB with transbronchial biopsy were not showed to predict bleeding [29-31]. Furthermore, animal data on a small number of anticoagulated pigs showed no bleeding [32], supporting the belief that life-threatening bleeding often results from the unpredictable biopsy of a sizeable pulmonary or bronchial artery [4]. Considering the paucity of data, the British Thoracic Society (BTS) recommends performing coagulation studies, a platelet count and haemoglobin concentration when there are clinical risk factors for abnormal coagulation (low level of evidence/grade D).

BAL can be performed with platelet counts greater than 20000 per $\mu \mathrm{L}$ (Grade D) [5]. Although not supported by published data, BAL is safely performed even in patients receiving a therapeutic anticoagulation or double antiplatelet treatment.

Uraemia affects platelet function and prolongs bleeding time. There is a paucity of data on the safety of FB in uraemic patients. An increased risk of bleeding with TBLB [1,22] could not be documented in a recent retrospective study [33]. Patients on chronic haemodialysis received haemodialysis and desmopressin intravenously before the procedure, and some authors suggest this approach [34]. Most pulmonologists, however, do not consider a high creatinine value as a contraindication to perform TBLB, and only $37 \%$ of them consider it appropriate to administer desmopressin prior to TBLB in uraemic patients [26]. Liver insufficiency and failure may induce coagulation abnormalities (thrombin time prolongation and thrombocytopenia), which may become clinically significant. In the suspicion, or evidence, of liver disease, we suggest performing coagulation studies and to optimise the coagulation status prior to bronchoscopy. 
A large number of drugs inhibiting platelet function or coagulation, with very different pharmacodynamic and pharmacokinetic properties, are registered for several indications and many more are released every year. Inevitably, these drugs raise the risk of iatrogenic bleeding, and the indication for tissue sampling as well as the risk and effectiveness of withholding antithrombotic treatment should be discussed on a case-by-case basis, considering and balancing the risk of thrombotic complications and the potential benefit from tissue sampling. The discontinuation of or alternatives to anticoagulation or antiplatelet therapy should be evaluated and discussed carefully with the treating cardiologist, neurologist or angiologist. For a few of these drugs, well-designed studies have shown their influence on iatrogenic bleeding risk during bronchoscopy [8, 35]. Although, for many antithrombotic drugs (e.g. ticagrelor, prasugrel, rivaroxaban and apixaban), no data are available specifically for bronchoscopy, the potential risk of bleeding may be extrapolated from the incidence of major bleeding reported in registry trials [36, 37]. Acetylsalicylic acid has been shown not to increase the risk of bleeding in patients undergoing TBLB [8], while clopidogrel has been associated with a significant increase in the frequency of clinically significant bleeding after TBLB [35]. In fact, this landmark trial was prematurely stopped because of an excessively high rate of bleeding in the clopidogrel group. An unpublished trial including only 15 patients and EBUS-TBNA of 40 lymph nodes showed a low risk of bleeding in patients taking clopidogrel [14]. Considering the pharmacokinetic properties of clopidogrel, we suggest withholding the drug for 7-10 days before performing TBLB and other invasive procedures. Prasugrel [38] and ticagrelor are newly developed adenosine diphosphate (ADP) receptor antagonists that induce higher and more consistent levels of platelet inhibition than clopidogrel [39]. These drugs have been associated with an increased rate of spontaneous major bleeding and should therefore be withheld before performing invasive bronchoscopic interventions.

\section{Patients receiving therapeutic anticoagulation}

For patients under therapeutic anticoagulation with LMWHs or direct antithrombin inhibitors, and in whom this treatment may be withheld for a short period of time, the suggested time prior to FB and tissue sampling is reported in table 1 . As previously mentioned, as the risk of a thromboembolic event (TE) increases, it is critical to minimise or discontinue anticoagulation therapy for as short a period as possible. The recommendation to perform a periprocedural intravenous UFH or subcutaneous LMWH bridging therapy depends on the risk of TE: it is not necessary in low-risk situations, but it can be considered in intermediate-risk situations and should be administered in high-risk scenarios [5, 40]. For most patients presenting a low to intermediate risk of TE (no mechanical heart valve; absence of stroke or systemic embolism within the previous 12 weeks; cardioembolic risk score (CHADS2) $<5$ points) stopping warfarin 5 days prior to bronchoscopy and resuming it the evening after the procedure is the approach of choice [5] and is not associated with an increased risk of TE [41].

For patients at high risk for a thrombotic complication (e.g. patients with a recent pulmonary embolism), it is preferable to perform bronchoscopy and tissue sampling at least 3 months after the PE or perform the tissue sampling with a periprocedural UFH or LMWH bridging therapy (as described above).

\section{Risk factors related to the tissue sampled}

The vascularisation of the tissue sampled is a significant risk factor for bleeding. Biopsies of hypervascularised inflammatory tissue, tumours or metastasis are associated with a higher rate of clinically significant bleeding. Several publications have reported a higher frequency of severe bleeding after endoscopic biopsy of carcinoids [42]. Dixon et al. [42] reported a retrospective analysis of all bronchopulmonary carcinoid tumours referred to a tertiary-care facility over one decade. Thirty patients underwent bronchoscopy for diagnostic evaluation, and only two complications of moderate to severe bleeding occurred, with no emergent thoracotomies, transfusions or deaths. Endobronchial metastasis of renal cancers or thyroid cancers, amyloidosis or bronchial anthracosis are also more prone to severe haemorrhage.

Furthermore, a situation associated with a very high risk for severe bleeding is the presence of vascular abnormalities. Biopsy of hypertrophic dysplastic arteries running in the bronchial submucosa, which may appear as a non-pulsating endobronchial lesion, vascular abnormalities in patients with bronchiectasis or pulmonary artery pseudoaneurysm induced by endobronchial brachytherapy may causes massive iatrogenic bleeding $[43,44]$.

\section{Prophylactic measures}

The indication for flexible bronchoscopy and sampling procedures as well as the potential risks and benefits should always be carefully evaluated. In particular, the necessity to perform TBLB must be dictated by potentially important clinical implications. Resuscitation equipment and additional staff necessary for emergency resuscitation should be readily available should an unexpected life-threatening 
TABLE 1 Suggested cutoff values for several parameters and time interval for the interruption of different drugs prior to the most commonly performed procedures during flexible bronchoscopy

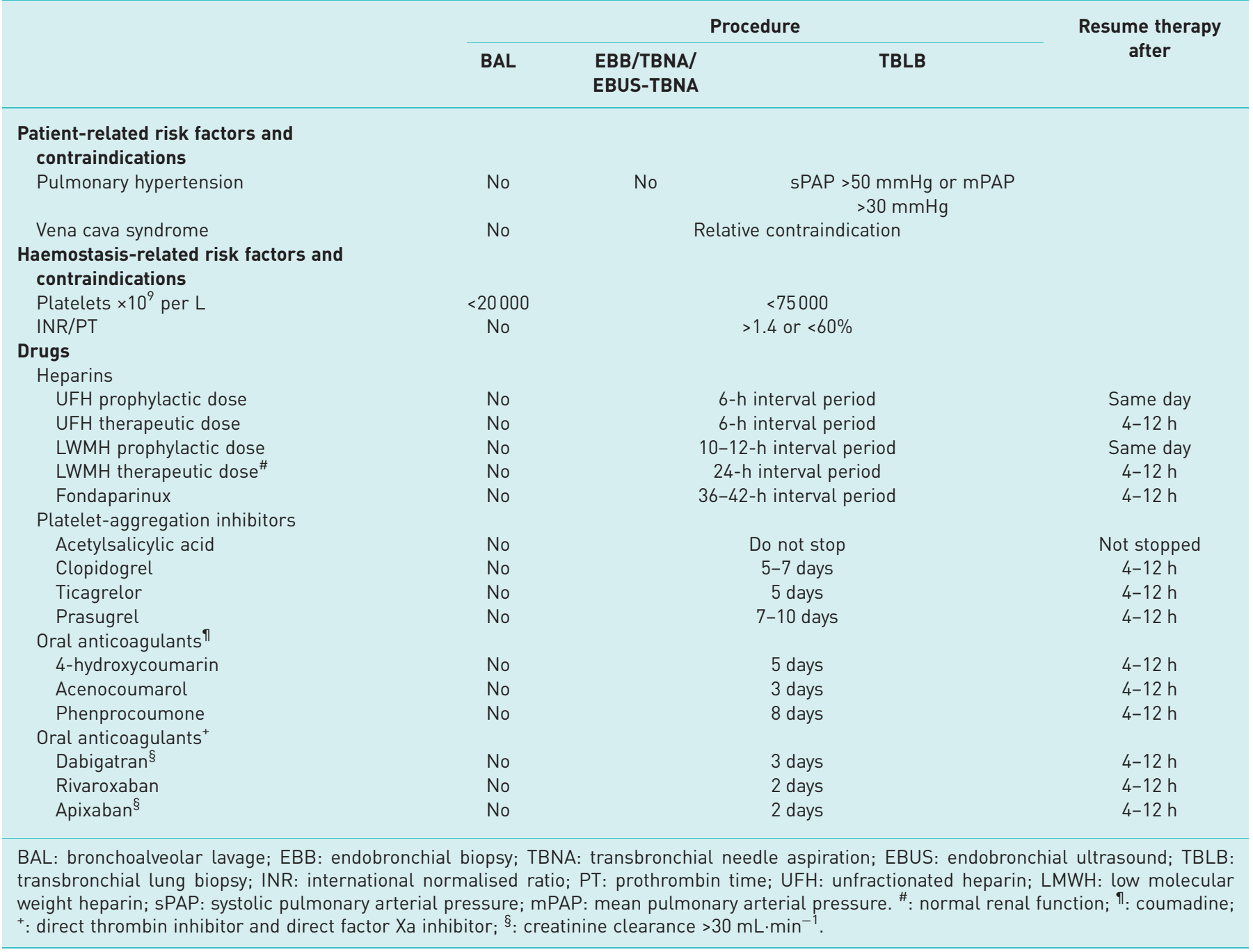

complication such as massive bleeding occur. Potentially correctable risk factors (figure 1) should be identified and optimised prior to performing FB [5].

For bronchoscopy through the nares, phenylephrine $0.5 \%$ or xylometazoline $0.1 \%$ can be helpful in vasoconstricting the mucosa of the nares and allowing easier passage of the scope.

The role of the prophylactic instillation of vasoconstrictors in the airways, mostly adrenaline (epinephrine) or terlipressin, is not well studied. The BTS recommend prophylactic instillation if bleeding is likely [5]. Adverse cardiac events, however, can occur at doses as low as $100 \mu \mathrm{g}$ of adrenaline [45], and the effects of adrenaline in the airways may be unpredictable. The instillation of vasoconstrictors is widely recommended for well-vascularised endobronchial tumours (bleeding from a central lesion). No data are available about the value of this practice before TBLB of peripheral lung lesions or in diffuse parenchymal lung disease (bleeding from the periphery). In cases at high risk for bleeding, the use of a bronchoscope with a large working channel should be considered.

TBLB is the procedure most commonly associated with complications, and life-threatening bleeding often results from the unpredictable biopsy of a sizeable pulmonary or bronchial artery [4]. TBLBs may be performed for a localised peripheral lesion or diffuse parenchymal pulmonary disease. In the latter, sampling of dependent segments may facilitate self-tamponade as well as limit inundation of the bronchial tree in the case of bleeding, helping to maintain visualisation. In fact, further visualisation is crucial in order to apply endobronchial therapies directed to stop the bleeding. Two different TBLBs techniques have been described. The most commonly recommended [5] techniques are 1) to wedge the bronchoscope into 
the target airway during and after the biopsy until haemostasis is achieved, avoiding suctioning in order to allow clot stabilisation [46], or 2) without wedging the bronchoscope, suction with the "back-and-forth technique" in the case of bleeding until bleeding stops [24]. These two techniques have not been compared regarding the frequency and efficacy of bleeding control.

\section{Plan of action in the case of bleeding} General considerations

Significant iatrogenic endobronchial bleeding is rare and mostly stops without further intervention. However, serious bleeding occasionally occurs and may be associated with significant morbidity. It is suggested that every bronchoscopy unit should have a standard operating procedure (SOP) or action plan in case of significant bleeding in order to optimise care and reduce morbidity.

When bleeding occurs, it is paramount to differentiate between a limited, self-controlled bleed and clinically significant bleeding requiring intervention. In fact, the signs and features of clinically significant and potentially life-threatening bleeding should prompt interventions or even the involvement of a resuscitation team, as in the case of massive haemoptysis. Similarly to the definition of massive haemoptysis [47], the term "life-threatening bleeding" may provide a more broad and informative description of the risk and the kind of therapeutic response needed. The consequences of an iatrogenic bleeding go beyond actual blood loss. The lung function and gas exchange prior to the intervention as well as the ability to maintain the airways clear of blood, which is often reduced due to the concomitantly administered sedation, are important factors to be considered.

\section{Plan of action for life-threatening iatrogenic bleeding}

Massive bleeding, persistent desaturation and haemodynamic instability should be viewed as warning signs requiring the assistance of a resuscitation team (figure 2). The management strategies used in this case are strongly dependent on local expertise. Experienced centres often favour an assertive approach with general anaesthesia (including mechanical and the application of positive end-expiratory pressure (PEEP)), rigid bronchoscopy and advanced haemostatic therapies that allow the rapid clearance of blood from the inundated bronchial tree, isolation of the bleeding lung/lobe with ventilation/oxygenation of the other non-bleeding lobes, and advanced haemostatic therapies for bleeding control. Advance haemostatic therapies include argon plasma coagulation and laser coagulation of the central bleeding source, and insertion of packing or blocking devices in the case of peripheral bleeding. However, rapid sequence orotracheal intubation, mechanical positive pressure ventilation with the application of PEEP and introduction of a bronchoscope with a large working channel scope and a haemostatic balloon next to it are a valuable alternative. Further, selective intubation and exclusion of the bleeding lung can also be considered. After stabilisation bronchial artery embolisation may be considered.

\section{Plan of action for a non-life-threatening iatrogenic bleeding}

In the case of non-hypoxaemic bleeding, we suggest differentiating it according to the source of bleeding, as specific advanced manoeuvres for the control of bleeding differ slightly depending on the site of bleeding; i.e. bleeding from a central endobronchial lesions (e.g. after an endobronchial biopsy of an endobronchial tumour) versus bleeding from the lung periphery (e.g. after transbronchial biopsies of a peripheral lesion or lung parenchyma).

\section{Bleeding from the lung periphery}

The plan of action for bleeding from the periphery (e.g. after transbronchial biopsy of lung tissue or peripheral lesion) proposed in figure 2 has been developed by integrating the available literature, as well as local experience and expertise. The recommended management approach has not been validated prospectively and may not be applicable in all centres, because it is largely influenced by the technique used to perform TBLB (wedging technique versus non-wedging technique), local expertise and available resources. Despite these limitations, it represents the plan of action we consider the most appropriate.

Turning the patient onto the bleeding side and in an anti-Trendelenburg position should be the first reaction in order to prevent and minimise inundation of the non-bleeding lung and guarantee adequate oxygenation.

Even if it has never been compared in a trial assessing the frequency and risk of bleeding, the wedging technique for TBLB [46] has the advantages of allowing endoluminal compression and blood stasis (which promotes clot formation and haemostasis) without losing the position, which could be difficult to regain in the case of diminished visibility. For these reasons, we consider this technique to be the technique of choice and recommend applying suction only 2-3 min after the TBLB has been performed in order to allow clot formation. Differently, continuous suction should be applied where visibility has been lost, in 


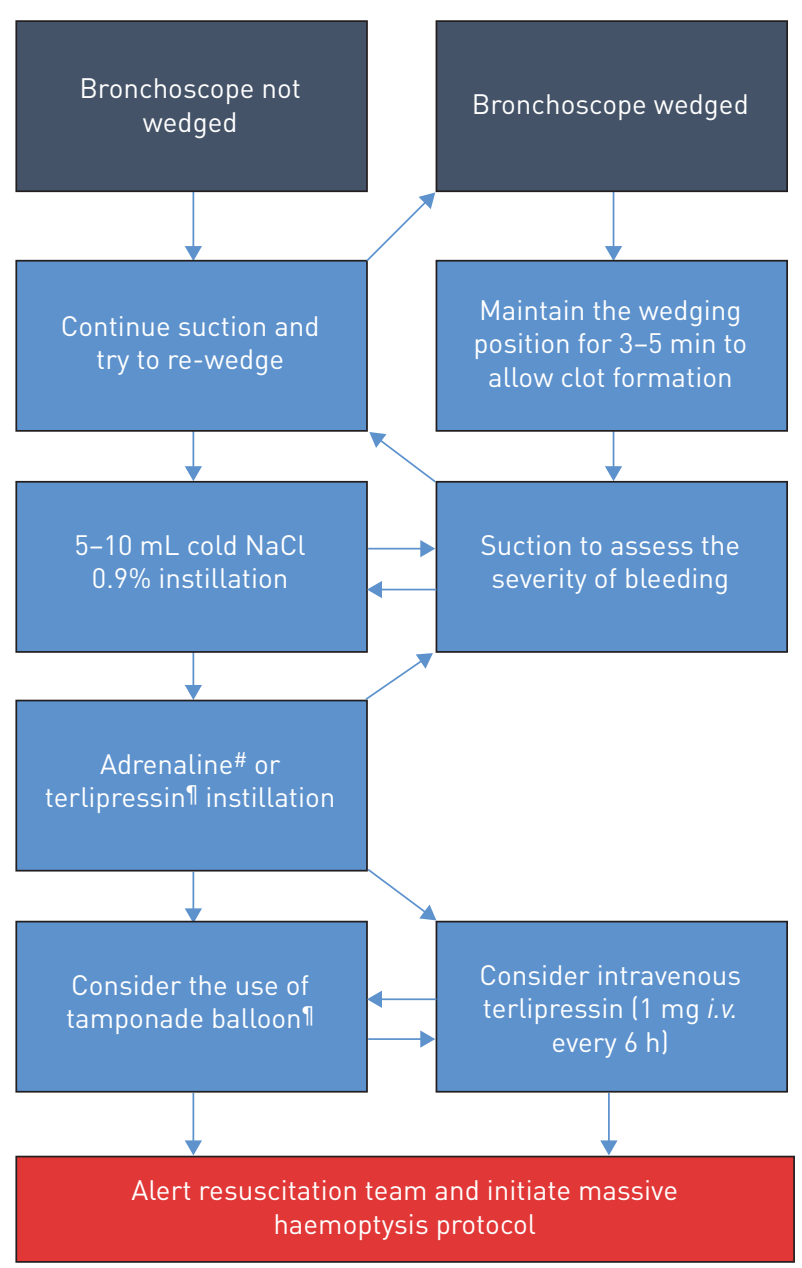

Reposition the patient in order to have the bleeding lung in the dependent position and the patient in anti-Trendelenburg position

Alert resuscitation team in case of:

Massive bleeding

Desaturation

Haemodynamic instability

FIGURE 2 Proposed plan of action in the case of iatrogenic bleeding during flexible bronchoscopy originating from the periphery. \# : adrenaline solution f.e. 1:25000=0.04 $\mathrm{mg} \cdot \mathrm{mL}^{-1}=40 \mu \mathrm{g} \cdot \mathrm{mL}^{-1}$. Maximum volume allowed for instillation: $1 \mathrm{~mL} / 10 \mathrm{~kg}$ body weight (e.g. $7 \mathrm{~mL}$ for $70 \mathrm{~kg}$ of body weight). ๆ: terlipressin (glypressin) $0.2 \mathrm{mg} \cdot \mathrm{mL}^{-1}$, e.g. $2 \mathrm{~mL}$, to be repeated if needed.

order to maintain airway clearance and visibility as well as regain the wedging position in the bleeding segment/subsegment.

The instillation of ice-cold saline and the use of vasoconstrictors and procoagulants have been suggested to promote haemostasis in the setting of endobronchial bleeding. There are limited reports, however, describing clinical outcomes that can convincingly advocate for or against these procedures. Ice-cold $0.9 \%$ saline instillation is often the first measure adopted, originating from the concept of cold-induced vasoconstriction, which, similar to cutaneous vessels [48], could occur in pulmonary vessels after localised cold exposure. Furthermore, the instillation of a certain volume of saline has the advantage of helping cleaning the optic and regain visibility. Hypothermic inhibition of clot formation [49] is not expected to play a significant role after repeated instillation of 5-10 $\mathrm{mL}$ of ice-cold saline.

The use of vasoconstrictors, especially as instillation, is also common practice in the case of iatrogenic bleeding [5]. As pulmonary organogenesis occurs by branching morphogenesis, proximal administration and absorption of vasoconstrictors may constrict the vessels feeding the injured vasculature, thus decreasing flow and promoting haemostasis. However, the contribution to haemostasis made by vasoconstrictors in patients with severe bleeding following TBLB is unknown, and no vasoconstrictor has been reported to be more effective than another. Adrenaline has been, by far, the most exhaustively studied and used substance. Despite that, the doses and dilutions of adrenaline suggested by textbooks, publications and guidelines for management of airway bleeding during bronchoscopy vary widely from "small amounts" of 1:100000 solution up to $20 \mathrm{~mL}$ of 1:20000 solution, thus leading to some confusion [50]. The BTS guidelines propose the instillation of 5-10 mL of 1:10000 adrenaline (500-1000 $\mu \mathrm{g}$ ) [5]. Cardiac arrhythmia after adrenaline instillation has been well known to be a complication since the 1970s [45], 
and adverse cardiac events can occur at doses as low as $100 \mu \mathrm{g}$. Limited data are available on the systemic absorption and effects of endobronchially administered adrenaline. In patients undergoing elective hip surgery $0.5 \mu \mathrm{g} \cdot \mathrm{kg}^{-1}$ endotracheal adrenaline had no haemodynamic effects [51]. Recently, potentially fatal arrhythmia occurred after the instillation of $5 \mathrm{~mL}$ of 1:20000 diluted adrenaline $(200 \mu \mathrm{g})$ via the guide sheath of a radial probe endobronchial ultrasound (R-EBUS) in two patients developing iatrogenic bleeding after TBLB [52], suggesting that the site of instillation may play a role. This theory is supported by a pharmacokinetic study in a canine model that demonstrated a more than fourfold increase in plasma levels when adrenaline was directly administered into the distal versus the proximal airways [53]. The antidiuretic hormone (ADH or vasopressin) analogues terlipressin (also called glypressin) and ornipressin have potent effects on plasma osmolality, volume and vascular tone acting on V1, V2, V3 and oxytocin-type receptors (OTRs). V1 receptors are found on vascular smooth muscle of the systemic, splanchnic, renal and coronary circulations. The haemostatic effect of ADH analogues is most likely due to the potent bronchial artery vasoconstriction combined with vasopressin-induced pulmonary vasodilation [54]. Intravenous application of terlipressin in doses typically administered for oesophageal variceal bleeding $(2 \mathrm{mg} / 4 \mathrm{~h})$ [55] has been shown to stop or significantly reduce the bleeding in patients with severe haemoptysis of different origins [56]. Furthermore, the instillation of $0.5 \mathrm{mg}$ terlipressin in the airways has been associated with bleeding stop and minor haemodynamic changes in a retrospective study comparing terlipressin to ornipressin in patients developing biopsy-associated bleeding [57]. Even if the plasma level after intravenous terlipressin administration was 251-fold higher than after endobronchial administration, the bronchial mucosa pallor appeared earlier after topical than after intravenous terlipressin application [58]. Unfortunately, terlipressin in combination or versus adrenaline has never been studied. As reported in table 2, after the instillation of adrenaline and in the case of bleeding persistence, and as a temporary measure awaiting the intervention of the resuscitation team and the interventional pulmonologist, we propose considering the administration of intravenous terlipressin.

Tranexamic acid (TXA) is an antifibrinolytic agent that acts by binding to plasminogen and blocking the interaction of plasminogen with fibrin, thereby preventing dissolution of the fibrin clot [59]. The very encouraging results of the CRASH-2 trial showing a significant mortality reduction in bleeding adult trauma patients [60] suggested the administration of this drug for other bleeding situations. The rationale behind the positive effect of TXA in bleeding trauma patients is that the stimulation of fibrinolysis due to trauma may become pathological (hyper-fibrinolysis) and promote bleeding [61]. In a pilot not-controlled study including iatrogenic and non-iatrogenic endobronchial bleeding, the instillation of TXA was shown to be a promising technique, especially for iatrogenic bleeding [62]. Other case studies revealed similar results $[63,64]$. However, thrombotic complications after the administration of TXA have been reported. Considering the lack of evidence regarding TXA administration for endobronchial bleeding and the potentially serious side events (thrombotic complications), we do not recommend its use in this situation $[65,66]$.

Fibrin sealant, also called fibrin glue, is a two-component system in which a solution of concentrated fibrinogen and factor XIII is combined with a solution of thrombin and calcium in order to form a coagulum, simulating the final stage of the clotting cascade. It is mostly applied as liquid glue or a sealant patch in the case of surgery-induced bleeding. Several case reports have described the successful instillation of fibrin sealant for persistent severe haemoptysis [67-69], without any complications.

TABLE 2 Summary of level of evidence and grade of recommendation as suggested in the British Thoracic Society guidelines [5]

\begin{tabular}{|c|c|c|c|}
\hline Procedure & $\begin{array}{l}\text { Level of } \\
\text { evidence }\end{array}$ & $\begin{array}{l}\text { Grade of } \\
\text { recommendation }\end{array}$ & Ref. \\
\hline $\begin{array}{l}\text { Perform coagulation studies, platelet count and haemoglobin concentration when there } \\
\text { are clinical risk factors for abnormal coagulation }\end{array}$ & 3 & D & [5] \\
\hline $\begin{array}{l}\text { LTX recipiens are more likely to bleed after TBLBs (not explained by clotting values or } \\
\text { aspirin use) }\end{array}$ & 3 & & [24] \\
\hline $\begin{array}{l}\text { Inspection and BAL can be performed in severely thrombocytopenic patients ( platelet } \\
>20000 \times 10^{9} \text { per } L^{-1} \text { ) }\end{array}$ & 3 & D & [10] \\
\hline $\begin{array}{l}\text { Clopidogrel has been associated with a significant increase in the frequency of clinically } \\
\text { significant bleeding after TBLB }\end{array}$ & 2 & C & [35] \\
\hline
\end{tabular}

TBLB: transbronchial lung biopsy; EBB: endobronchial biopsy; LTX: lung transplant; BAL: bronchoalveolar lavage. 


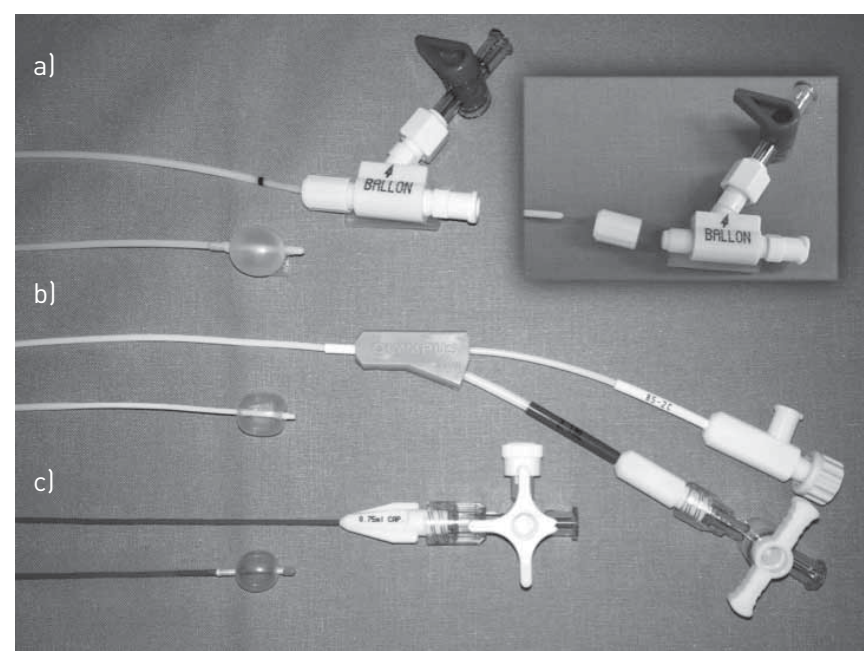

FIGURE 3 Different types of haemostatic balloon catheters. a) Decomposable haemostatic endoscopic balloon catheter (size 6 French $(2 \mathrm{~mm}$ ), minimum working channel size $2.4 \mathrm{~mm}$ ) allowing placement of the catheter through the working channel and removal of the bronchoscope without removal of the catheter (Rüsch Bronchus Blocker; Teleflex Medical, Kernen, Germany). b) Haemostatic endoscopic balloon catheter (size 4 French $(1.4 \mathrm{~mm})$, minimum working channel size $2 \mathrm{~mm}$, maximal diameter of the inflated balloon $11 \mathrm{~mm})$ for blocking lobar or segmental bronchi (haemostatic balloon catheter; Olympus, Tokyo, Japan). The blockage of a main bronchus can be achieved with the larger Arndt and Cohen endobronchial blockers (7-9 French, Cook Medical, Bloomington, IN, USA). c) Fogarty arterial embolectomy catheter (size 4 French (1.35 mm), minimum working channel size $2 \mathrm{~mm}$ ). Fogarty catheters are available in sizes ranging from 2 to 8 French.

Furthermore, the use of different types of tamponade balloons has been described (figure 3). Fogarty balloons (Fogarty Occlusion Catheter; Edwards Lifesciences, Irvine, CA, USA) may have the advantage of being universally available and exist in a wide range of sizes. Specifically developed tamponade balloons are double-lumen, bronchus-blocking catheters that can be introduced through the working channel of a standard flexible bronchoscope to be placed into the bleeding bronchial segment. Moreover, the internal lumen allows the instillation of substances improving haemostasis. The successful use of this balloon has been reported in 26 patients with moderate to severe bleeding due to different conditions [70]. Balloons were placed in the lobar or segmental bronchi and left up, usually between a few hours and 2-3 days (up to 7 days), allowing stabilisation and a complementary treatment (surgery, radiation or bronchial arterial embolisation). Larger balloons with larger catheters may be introduced through an endobronchial tube next to the scope and block a main bronchus, staying in place via a three-way port after the scope is retracted.

\section{Bleeding from a central lesion}

The principles of bleeding management originating from a central lesion do not differ significantly from that applied in a bleeding originating from a peripheral lesion. Similarly, a haemostatic balloon may be used to encourage local coagulation. However, with a visible central source of bleeding, the opportunity for targeted coagulation should be considered. Argon plasma coagulation (APC) is a noncontact thermal ablation technique that uses argon gas to generate heat, which, in turn, can be used to achieve haemostasis $[71,72]$. Thermal energy is delivered with a depth of penetration of roughly $2-3 \mathrm{~mm}$, producing tissue coagulation and destruction. Due to these features, APC is a useful modality for the treatment of superficial/flat lesions, as well as those that are highly vascular or bleeding. Its advantages include its ease of application and safety due to its reduced depth of penetration and lower cost compared to laser techniques. The plasma jet also improves visualisation of the bleeding lesion due to the clearing effect of the gas jet. Laser coagulation therapies (mostly Nd:YAP or diode lasers) are other valuable possibilities that allow deeper coagulation. However, laser coagulation should be performed by an experienced interventional bronchoscopist due to the deeper penetration and risk of complications [73]. Furthermore, monopolar electrocautery can be used for contact diathermy coagulation.

In conclusion, significant iatrogenic bleeding during FB is rare and usually self-limiting. Life-threatening bleeding may occur, especially after conventional or cryoprobe-assisted TBLB. Bronchoscopy units should have a standardised approach and plan of action in case of life-threatening haemorrhage, including the involvement of a resuscitation team. Wedging the bronchoscope in the bleeding segment, instillations of vasoconstrictors and the early use of a tamponade balloon are the recommended first-line strategies. 


\section{References}

1 Cordasco EM, Mehta AC, Ahmad M. Bronchoscopically induced bleeding. A summary of nine years' Cleveland clinic experience and review of the literature. Chest 1991; 100: 1141-1147.

2 Chinsky K. Bleeding risk and bronchoscopy: in search of the evidence in evidence-based medicine. Chest 2005 127: $1875-1877$.

3 Zhou G-W, Zhang W, Dong Y-C, et al. Flexible bronchoscopy-induced massive bleeding: a 12-year multicentre retrospective cohort study. Respirology 2016; 21: 927-931.

4 Flick MR, Wasson K, Dunn LJ, et al. Fatal pulmonary hemorrhage after transbronchial lung biopsy through the fiberoptic bronchoscope. Am Rev Respir Dis 1975; 111: 853-856.

5 Du Rand IA, Blaikley J, Booton R, et al. British Thoracic Society guideline for diagnostic flexible bronchoscopy in adults: accredited by NICE. Thorax 2013; 68: Suppl. 1, i1-i44.

6 Facciolongo N, Patelli M, Gasparini S, et al. Incidence of complications in bronchoscopy. Multicentre prospective study of 20,986 bronchoscopies. Monaldi Arch Chest Dis 2009; 71: 8-14.

7 Milman N, Faurschou P, Munch EP, et al. Transbronchial lung biopsy through the fibre optic bronchoscope. Results and complications in 452 examinations. Respir Med 1994; 88: 749-753.

8 Herth FJF, Becker HD, Ernst A. Aspirin does not increase bleeding complications after transbronchial biopsy. Chest 2002; 122: 1461-1464.

9 Trosini-Désert V, Vergnon JM, Collet JP, et al. Bronchoscopie souple et antiagrégants plaquettaires: analyse du rapport bénéfices-risques [Fibreoptic bronchoscopy and anti-platelet agents: a risk-benefit analysis]. Rev Mal Respir 24: 48-56.

10 Weiss SM, Hert RC, Gianola FJ, et al. Complications of fiberoptic bronchoscopy in thrombocytopenic patients Chest 1993; 104: 1025-1028.

11 Nandagopal L, Veeraputhiran $\mathrm{M}$, Jain $\mathrm{T}$, et al. Bronchoscopy can be done safely in patients with thrombocytopenia. Transfusion 2016; 56: 344-348.

12 Varela-Lema L, Fernández-Villar A, Ruano-Ravina A. Effectiveness and safety of endobronchial ultrasound-transbronchial needle aspiration: a systematic review. Eur Respir J 2009; 33: 1156-1164.

13 Eapen GA, Shah AM, Lei X, et al. Complications, consequences, and practice patterns of endobronchia ultrasound-guided transbronchial needle aspiration: results of the AQuIRE registry. Chest 2013; 143: 1044-1053.

14 Stather DR, MacEachern P, Chee A, et al. Safety of endobronchial ultrasound-guided transbronchial needle aspiration for patients taking clopidogrel: a report of 12 consecutive cases. Respiration 2012; 83: 330-334.

15 Ahmad M, Livingston DR, Golish JA, et al. The safety of outpatient transbronchial biopsy. Chest 1986; 90: 403-405.

16 Pue CA, Pacht ER. Complications of fiberoptic bronchoscopy at a university hospital. Chest 1995; 107: 430-432.

17 Hetzel J, Eberhardt R, Herth FJF, et al. Cryobiopsy increases the diagnostic yield of endobronchial biopsy: a multicentre trial. Eur Respir J 2012; 39: 685-690.

18 Tomassetti S, Wells AU, Costabel U, et al. Bronchoscopic lung cryobiopsy increases diagnostic confidence in the multidisciplinary diagnosis of idiopathic pulmonary fibrosis. Am J Respir Crit Care Med 2016; 193: 745-752.

19 Casoni GL, Tomassetti S, Cavazza A, et al. Transbronchial lung cryobiopsy in the diagnosis of fibrotic interstitial lung diseases. PLoS One 2014; 9: e86716.

20 Schuhmann M, Bostanci K, Bugalho A, et al. Endobronchial ultrasound-guided cryobiopsies in peripheral pulmonary lesions: a feasibility study. Eur Respir J 2014; 43: 233-239.

21 Pajares V, Puzo C, Castillo D, et al. Diagnostic yield of transbronchial cryobiopsy in interstitial lung disease: a randomized trial. Respirology 2014; 19: 900-906.

22 Cunningham JH, Zavala DC, Corry RJ, et al. Trephine air drill, bronchial brush, and fiberoptic transbronchial lung biopsies in immunosuppressed patients. Am Rev Respir Dis 1977; 115: 213-220.

23 Diette GB, Wiener CM, White P. The higher risk of bleeding in lung transplant recipients from bronchoscopy is independent of traditional bleeding risks: results of a prospective cohort study. Chest 1999; 115: 397-402.

24 Chhajed PN, Aboyoun C, Malouf MA, et al. Risk factors and management of bleeding associated with transbronchial lung biopsy in lung transplant recipients. J Heart Lung Transplant 2003; 22: 195-197.

25 Brundyn K, Koegelenberg CFN, Diacon AH, et al. Transbronchial fine needle aspiration biopsy and rapid on-site evaluation in the setting of superior vena cava syndrome. Diagn Cytopathol 2013; 41: 324-329.

26 Wahidi MM, Rocha AT, Hollingsworth JW, et al. Contraindications and safety of transbronchial lung biopsy via flexible bronchoscopy. A survey of pulmonologists and review of the literature. Respiration 2005; 72: 285-295

27 Diaz-Guzman E, Vadi S, Minai OA, et al. Safety of diagnostic bronchoscopy in patients with pulmonary hypertension. Respiration 2009; 77: 292-297.

28 Committee on Standards and Practice Parameters et al. Practice advisory for preanesthesia evaluation: an updated report by the American Society of Anesthesiologists Task Force on Preanesthesia Evaluation. Anesthesiology 2012; 116: 522-538.

29 Kozak EA, Brath LK. Do 'screening' coagulation tests predict bleeding in patients undergoing fiberoptic bronchoscopy with biopsy? Chest 1994; 106: 703-705.

30 Bjørtuft O, Brosstad F, Boe J. Bronchoscopy with transbronchial biopsies: measurement of bleeding volume and evaluation of the predictive value of coagulation tests. Eur Respir J 1998; 12: 1025-1027.

31 Carr IM, Koegelenberg CFN, von Groote-Bidlingmaier F, et al. Blood loss during flexible bronchoscopy: a prospective observational study. Respiration 2012; 84: 312-318.

32 Brickey DA, Lawlor DP. Transbronchial biopsy in the presence of profound elevation of the international normalized ratio. Chest 1999; 115: 1667-1671.

33 Mehta NL. Should renal insufficiency be a relative contraindication to bronchoscopic biopsy? J Bronchol Interv Pulmonol 2005; 12: 81-83.

34 Khan I, Bellinger C, Lamb C, et al. Bronchoscopy in uremic patients. Clin Pulmonary Med 2010; 17: 146-148.

35 Ernst A, Eberhardt R, Wahidi $\mathrm{M}$, et al. Effect of routine clopidogrel use on bleeding complications after transbronchial biopsy in humans. Chest 2006; 129: 734-737.

36 Serebruany VL, Malinin AI, Eisert RM, et al. Risk of bleeding complications with antiplatelet agents: meta-analysis of 338,191 patients enrolled in 50 randomized controlled trials. Am J Hematol 2004; 75: 40-47.

37 Chatterjee S, Sardar P, Biondi-Zoccai G, et al. New oral anticoagulants and the risk of intracranial hemorrhage: traditional and Bayesian meta-analysis and mixed treatment comparison of randomized trials of new oral anticoagulants in atrial fibrillation. JAMA Neurol 2013; 70: 1486-1490. 
38 Wiviott SD, Antman EM, Braunwald E. Prasugrel. Circulation 2010; 122: 394-403.

39 Guimarães PO, Tricoci P. Ticagrelor, prasugrel, or clopidogrel in ST-segment elevation myocardial infarction: which one to choose? Expert Opin Pharmacother 2015; 16: 1983-1995.

40 Jakubec P, Kolek V, Rakita D, et al. Expert consensus statement for periprocedural anticoagulation and antiplatelet therapy in elective bronchoscopy. Cor et Vasa 2016; 58: e175-e180.

41 Douketis JD, Spyropoulos AC, Kaatz S, et al. Perioperative bridging anticoagulation in patients with atrial fibrillation. N Engl J Med 2015; 373: 823-833.

42 Dixon RK, Britt EJ, Netzer GA, et al. Ten-year single center experience of pulmonary carcinoid tumors and diagnostic yield of bronchoscopic biopsy. Lung 2016; 194: 905-910.

43 Trisolini R, Cancellieri A, Patelli M. Life-threatening bleeding after endobronchial biopsy in a patient with bronchiectasis. Am J Respir Crit Care Med 2013; 188: e9-e10.

44 Chawla M, Getzen T, Simoff MJ. Medical pneumonectomy: interventional bronchoscopic and endovascular management of massive hemoptysis due to pulmonary artery pseudoaneurysm, a consequence of endobronchial brachytherapy. Chest 2009; 135: 1355-1358.

45 Mall W, Abel H. Topical application of epinephrine during bronchoscopy in barbiturate-halothane-anaesthesia and its influence on cardiac action. Bronchopneumologie 1978; 28: 311-316.

46 Zavala DC. Diagnostic fiberoptic bronchoscopy: techniques and results of biopsy in 600 patients. Chest 1975; 68 : 12-19.

47 Ibrahim WH. Massive haemoptysis: the definition should be revised. Eur Respir J 2008; 32: 1131-1132.

48 Bailey SR, Eid AH, Mitra S, et al. Rho kinase mediates cold-induced constriction of cutaneous arteries: role of

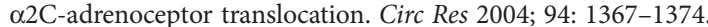

49 Whelihan MF, Kiankhooy A, Brummel-Ziedins KE. Thrombin generation and fibrin clot formation under hypothermic conditions: an in vitro evaluation of tissue factor initiated whole blood coagulation. J Crit Care 2014; 29: 24-30.

50 Khoo KL, Lee P, Mehta AC. Endobronchial epinephrine: confusion is in the air. Am J Respir Crit Care Med 2013; 187: 1137-1138

51 McCrirrick A, Kestin I. Haemodynamic effects of tracheal compared with intravenous adrenaline. Lancet 1992; 340: 868-870.

52 Steinfort DP, Herth FJF, Eberhardt R, et al. Potentially fatal arrhythmia complicating endobronchial epinephrine for control of iatrogenic bleeding. Am J Respir Crit Care Med 2012; 185: 1028-1030.

53 Mazkereth R, Paret G, Ezra D, et al. Epinephrine blood concentrations after peripheral bronchial versus endotracheal administration of epinephrine in dogs. Crit Care Med 1992; 20: 1582-1587.

54 Enomoto M, Pan J, Shifrin Y, et al. Age dependency of vasopressin pulmonary vasodilatory effect in rats. Pediatr Res 2014; 75: 315-321.

55 García-Pagán JC, Reverter E, Abraldes JG, et al. Acute variceal bleeding. Semin Respir Crit Care Med 2012; 33: 46-54.

56 Ramon P, Wallaert B, Derollez M, et al. Traitement des hémoptysies graves par la terlipressine. Etude de l'efficacité et de la tolérance du produit. [Treatment of severe hemoptysis with terlipressin. Study of the efficacy and tolerance of this product]. Rev Mal Respir 1989; 6: 365-368.

57 Tüller C, Tüller D, Tamm M, et al. Hemodynamic effects of endobronchial application of ornipressin versus terlipressin. Respiration 2004; 71: 397-401.

58 Breuer HW, Charchut S, Worth H, et al. Endobronchial versus intravenous application of the vasopressin derivative glypressin during diagnostic bronchoscopy. Eur Respir J 1989; 2: 225-228.

59 McCormack PL. Tranexamic acid: a review of its use in the treatment of hyperfibrinolysis. Drugs 2012; 72 : 585-617.

60 CRASH-2 Trial collaborators, Shakur H, Roberts I, Bautista R, et al. Effects of tranexamic acid on death, vascular occlusive events, and blood transfusion in trauma patients with significant haemorrhage (CRASH-2): a randomised, placebo-controlled trial. Lancet 2010; 376: 23-32.

61 Lawson JH, Murphy MP. Challenges for providing effective hemostasis in surgery and trauma. Semin Hematol 2004; 41: 55-64.

62 Márquez-Martín E, Vergara DG, Martín-Juan J, et al. Endobronchial administration of tranexamic acid for controlling pulmonary bleeding: a pilot study. J Bronchol Interv Pulmonol 2010; 17: 122-125.

63 Zamani A. Bronchoscopic intratumoral injection of tranexamic acid: a new technique for control of biopsy-induced bleeding. Blood Coagul Fibrinolysis 2011; 22: 440-442.

64 Solomonov A, Fruchter O, Zuckerman T, et al. Pulmonary hemorrhage: a novel mode of therapy. Respir Med 2009; 103: 1196-1200.

65 Levrat A, Gros A, Rugeri L, et al. Evaluation of rotation thrombelastography for the diagnosis of hyperfibrinolysis in trauma patients. Br J Anaesth 2008; 100: 792-797.

66 Theusinger OM, Wanner GA, Emmert MY, et al. Hyperfibrinolysis diagnosed by rotational thromboelastometry (ROTEM) is associated with higher mortality in patients with severe trauma. Anesth Analg 2011; 113: 1003-1012.

67 Pandya CM, Adam A, Jahania S, et al. Use of fibrin sealant glue as a treatment for massive hemoptysis. $J$ Bronchology Interv Pulmonol 2011; 18: 97-98.

68 Cuervo F, Giraldo LF, Vélez C, et al. Fibrinógeno-trombina como tratamiento puente en un caso de hemoptisis masiva [Fibrinogen-thrombin as bridge therapy in massive hemoptysis]. Biomedica 2013; 33: 28-30.

69 de Gracia J, la Rosa de D, Catalán E, et al. Use of endoscopic fibrinogen-thrombin in the treatment of severe hemoptysis. Respir Med 2003; 97: 790-795.

70 Freitag L, Tekolf E, Stamatis G, et al. Three years experience with a new balloon catheter for the management of haemoptysis. Eur Respir J 1994; 7: 2033-2037.

71 Bolliger CT, Sutedja TG, Strausz J, et al. Therapeutic bronchoscopy with immediate effect: laser, electrocautery, argon plasma coagulation and stents. Eur Respir J 2006; 27: 1258-1271.

72 Gompelmann D, Eberhardt R, Herth FJF. Interventional pulmonology procedures: an update. Panminerva Med 2013; 55: 121-129.

73 Bolliger CT, Sutedja TG, Strausz J, et al. Therapeutic bronchoscopy with immediate effect: laser, electrocautery, argon plasma coagulation and stents. Eur Respir J 2006; 27: 1258-1271. 